\title{
HIV-1 Integrase Sequence Variability in Antiretroviral Naïve Patients and in Triple-Class Experienced Patients Subsequently Treated with Raltegravir
}

\author{
Vici Varghese,, Tommy F. Liu, Soo-Yon Rhee,, Paolo Libiran,2 Christina Trevino, \\ W. Jeffrey Fessel, ${ }^{3}$ and Robert W. Shafer ${ }^{1}$
}

\begin{abstract}
Viruses were sequenced from 75 antiretroviral therapy (ARV)-naïve and from 75 ARV-treated patients who subsequently received a raltegravir-containing regimen. No major integrase inhibitor (INI)-resistance mutations were present in the 150 integrase (IN) sequences. Four ARV-naïve (5.3\%) and two ARV-treated patients (2.7\%) had one of the following minor INI-resistance mutations: L74M, E157Q, G163R, and R263K but there was no association between baseline raltegravir genotype and subsequent response to raltegravir treatment. We also combined our sequences with 4170 previously published group M IN sequences from INI-naïve patients to assess IN sequence variability and compared our findings with those of a study we performed in 2008 using data from 1563 patients. The number of polymorphic IN positions increased from $40 \%$ to $41 \%$ between the two studies. However, none of the major INI-resistance mutations was found to be polymorphic in either study and there were no significant changes in the prevalence of any of the minor INI-resistance mutations.
\end{abstract}

C RYSTALLOGRAPHIC, BIOCHEMICAL, AND MODELING DATA suggest that HIV-1 integrase (IN) inhibitors (INIs) bind to a highly conserved region in active site of the IN enzyme. ${ }^{1-4}$ Preliminary studies have shown that the IN mutations that most commonly decrease INI susceptibility do not occur as natural polymorphisms in viral sequences from INI-naive patients, $^{5-7}$ but that several accessory INI-resistance mutations do occur as natural IN polymorphisms. ${ }^{6}$

HIV-1 RT forms part of the HIV-1 pre-integration complex, making it plausible that the spectrum of mutations in IN from heavily treated patients would be different from those in antiretroviral therapy (ARV)-naïve patients. ${ }^{8} \mathrm{We}$ therefore chose to sequence viruses from ARV-naïve and ARV-experienced patients to compare the proportion of IN variants in these two patient groups. The heavily treated patients were selected from a group of patients that eventually received a raltegravir-containing regimen, thereby enabling us to also examine whether any pre-raltegravir accessory INI-resistance variants might be associated with an increased risk of raltegravir failure. We then combined our sequences with previously published group $M$ IN sequences to characterize the extent of diversity within group M HIV-1 isolates.

\section{Patients, Samples, and Sequencing}

Plasma samples were collected from 160 ARV-naïve and -treated HIV-1 infected patients undergoing genotypic resistance testing between 2002 and 2008 at the Stanford University Hospital Diagnostic Virology Laboratory. The ARV-treated patients were selected from a group of 82 patients from whom cryopreserved plasma samples were available and who received a raltegravir-containing maintenance or salvage therapy regimen within 3 years of viral sampling. The ARV-naïve patients were selected from a group of 78 patients with similar plasma HIV-1 RNA levels as the ARV-treated patients. All ARV-treated patients were triple-class experienced having received a median of four NRTIs, one NNRTI, and two PIs. PCR amplification and IN sequencing were successfully performed for 75 of $78(96 \%)$ and 75 of $82(91 \%)$ of the samples from ARV-naïve and ARV-treated patients, respectively.

One $\mathrm{ml}$ of cryopreserved plasma sample was ultracentrifuged for $30 \mathrm{~min}$, and RNA was extracted using the Roche Amplicor RNA extraction kit. Reverse-transcription and a first round of PCR was performed using One-Step RTPCR system (Invitrogen, Carlsbad, CA) with Superscript III RT and Platinum Taq enzyme mix. The first round of PCR

\footnotetext{
${ }^{1}$ Department of Medicine, and ${ }^{2}$ Diagnostic Virology Laboratory, Stanford University School of Medicine, Stanford, California

${ }^{3}$ Clinical Trials Unit, Kaiser-Permanente Medical Care Program-Northern California, San Francisco, California.
} 
amplified a 1055-bp product using primers IN4164F (CAC AAAGGAATTGGAGGAAATGAAC; HXB2 positions 41644188) and IN5219R (CCTAGTGGGATGTGTACTTCTGAAC; HXB2 positions 5219-5195). A second round of PCR amplified a 983-bp product using primers IN4195F (ATAAATTAGT CAGTGCTGGAATC; HXB2 positions 4195-4217) and IN5178R (GCTTTCATAGTGATGTCTATA; HXB2 positions 5178-5158). Sanger sequencing was performed using BigDye terminator chemistry (Applied Biosystems, Foster City, CA) with primers IN4655F (CTACAATCCCCAAAGTCAAG GAGT HXB2 positions 4655-4678), IN4735R (GCCTGAT CTCTTACCTGTCCTAT HXB2 4735-4713), IN4195F, and IN5178R. The sequencing products were run on a $3100 \mathrm{Ge}-$ netic analyzer (Applied Biosystems); sequence data was analyzed using Sequencher v4.8.

Of the 150 sequenced IN genes, 146 belonged to subtype B (bootstrap value $\geq 97 \%$ according to the REGA HIV-1 Subtypting Tool). ${ }^{9}$ The mean uncorrected nucleotide distance between each of the nucleotide sequences within each subtype was 0.044 for both the ARV-naïve and ARV-treated patients. A neighborjoining phylogenetic tree was constructed using the HKY85 substitution model with the distribution of substitutions across sites modeled using gamma distribution (Supplemental Fig. 1; see www.liebertonline.com/aid).

For 33 of the 160 samples, sequencing was performed in duplicate in two laboratories (the authors' research laboratory and the Stanford University Hospital Diagnostic Virology Laboratory). The sequence concordance was $99.2 \%$ for the 28,512 nucleotides of the 33 IN samples sequenced in duplicate. Of the 214 discordances, all but 17 were partial (i.e., one sequence contained a nucleotide mixture whereas the other contained a component of that mixture).

\section{Proportion of Sequences with INI-Associated Mutations in ARV-Naïve and Triple-Class Experienced Patients}

Each amino acid difference from consensus was categorized according its reported association with INI resistance: ${ }^{10}$ (i) Major INI-resistance mutations were defined as mutations that phenotypically decrease susceptibility to raltegravir or elvitegravir by 5 -fold or higher in the PhenoSense assay (Monogram Biosciences, South San Francisco, CA) and have been reported to be selected by one of these INIs in vitro or in vivo: T66IAK, E92Q, F121Y (solely in vitro), G140SA, Y143HCR, Q146P (solely in vitro), S147G, Q148KHR, and N155HS; (ii) minor INI-resistance mutations were defined as nonpolymorphic or minimally polymorphic mutations that reduce INI susceptibility $<5$-fold by themselves or that significantly contribute to resistance when they occur in combination with other mutations: H51Y, L74M, T97A, E138AK, S153Y, E157Q, G163RK, S230R, and R263K; (iii) minor INIaccessory mutations were defined as highly polymorphic mutations that have been reported to occur more frequently
Table 1. Proportions of Integrase Sequences with Mutations Associated with Integrase-Inhibitor (INI) Exposure or REDUCED SUSCEPTIBILITY

\begin{tabular}{|c|c|c|c|c|}
\hline Category & Mutation & $\begin{array}{c}A R V \text { naïve } \\
(\mathrm{n}=75)\end{array}$ & $\begin{array}{c}A R V \\
\text { treated } \\
(\mathrm{n}=75)\end{array}$ & $\mathrm{p}$ Value \\
\hline $\begin{array}{l}\text { Major INI-resistance } \\
\text { mutations }\end{array}$ & None & 0 & 0 & NA \\
\hline \multirow{4}{*}{$\begin{array}{l}\text { Minor INI-resistance } \\
\text { mutations }\end{array}$} & L74M & $1(1.3)$ & 0 & NS \\
\hline & E157Q & 0 & $1(1.3)$ & NS \\
\hline & G163R & $1(1.3)$ & 0 & NS \\
\hline & R263K & $2(2.7)$ & $1(1.3)$ & NS \\
\hline \multirow{7}{*}{$\begin{array}{l}\text { Minor INI-accessory } \\
\text { mutations }\end{array}$} & V151I & $7(9.3)$ & $1(1.3)$ & 0.07 \\
\hline & M154I & $4(5.3)$ & $6(8)$ & NS \\
\hline & M154L & $1(1.3)$ & $9(12)$ & 0.02 \\
\hline & V165I & $2(2.7)$ & 0 & NS \\
\hline & V201I & 21 (28) & $25(33.3)$ & NS \\
\hline & I203M & $3(4)$ & $5(6.7)$ & NS \\
\hline & S230N & $6(8)$ & $6(8)$ & NS \\
\hline
\end{tabular}

Major INI resistance mutations: Nonpolymorphic mutations that phenotypically decrease susceptibility to Raltegravir or Elvitegravir by 5-fold or higher, T66IAK, E92Q, F121Y, G140SA, Y143HCR, Q146P, S147G, Q148KHR, and N155HSP; Minor INI-resistance mutations: minimally polymorphic mutations that reduce INI susceptibility $<5$ fold by themselves or that are significantly associated with resistance in combination with other mutations, H51Y, L74V, T97A, E138AK, S153Y, E157Q, G163RK, S230R, and R263K; Minor INI-accessory mutations: typically polymorphic mutations which have been reported at least once to occur more frequently among INI-treated patients, V68VI, V151IA, M154IL, V201I, I203M, and S230N.

Percentages given in parenthesis; NA: not applicable; NS: not significant. The unusual mutations in nonpolymorphic sites associated with a minor INI mutations in ARV-naïve and ARV-treated sequences included H51Q $(1+0)$, L74I $(1+2)$, Q95H $(1+0)$, Q95N $(0+1), \mathrm{E} 138 \mathrm{D}(1+1)$, and S153P $(2+0)$.

among INI-treated than INI-naïve patients but which have not been shown to contribute to reduced INI susceptibility.

Table 1 shows the proportions of our 150 IN sequences with mutations associated with INI exposure or reduced susceptibility. No major INI-resistance mutations were present in the 150 sequenced IN genes. Four ARV-naïve (5.3\%) and two ARVtreated patients $(2.7 \%)$ had one minor INI-resistance mutation, including R263K in three patients, and L74M, G163R, and E157Q each in one patient. Seven highly polymorphic minor INI-accessory mutations including V151I, M154I, M154L, V165I, V201I, I203M, and S230N occurred commonly in both ARV-naïve and ARV-treated individuals. M154L occurred more commonly in ARV-treated compared with ARV-naïve patients (12.0\% vs $1.3 \%$; $p=0.02$; Fisher's Exact Test), a finding which has independently been reported earlier. Several unusual mutations at minor INI-resistance positions were present in small numbers of both groups of patients (Table 1, footnote).

FIG. 1. Distribution of variants among group M HV-1 integrase sequences. The consensus subtype B sequence is shown at the top of each 40 amino acid section. Beneath the consensus B sequence is the number of annotated sequences containing an unambiguous amino acid at the indicated position with the number of such sequence ranging from 3730 to 4435 . All variants reported at a level of $\geq 0.5 \%$ of sequences are indicated. Gray shading surrounds the central core domain residues. Boxes indicate the signature HHCC zinc-binding motif in the N-terminal domain and the DDE active site residues in the central core domain. Positions at which primary INI-resistance mutations for raltegravir and elvitegravir have been reported are indicated by " $\star$ ". Positions at which accessory INI-resistance mutations for raltegravir and elvitegravir have been reported are indicated by " + ". Positions at which INI-resistance mutations for other inhibitors have been reported are indicated by "•". 


\begin{tabular}{|c|c|c|c|c|c|c|c|}
\hline 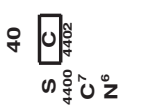 & 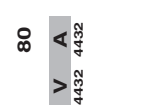 & 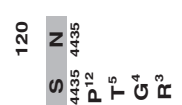 & 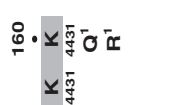 & 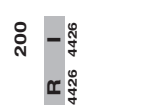 & 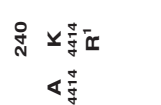 & 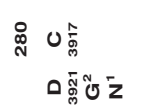 & \\
\hline 《券 & I孚 & 㧹 & 」离 & 山辣 & 唼 & 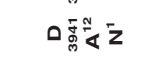 & \\
\hline 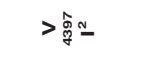 & $>$ 采 4 & z㝵 & 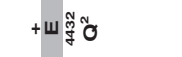 & 㗪 & 㖊 & ৩兽 & \\
\hline -兽 & 嫖 & 腹 & $x$ 草ž & $\varangle \stackrel{\dddot{g}}{q}$ & $x_{\frac{0}{q}}^{0}$ & 㢵 & \\
\hline ш患 & $>$ 孚 & r尊 & *z栾 & 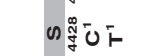 & $3 \frac{0}{4}$ & $\sum \frac{q}{n} \bar{c}$ & \\
\hline 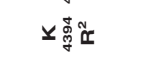 & 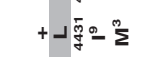 & I尊 & 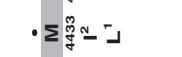 & 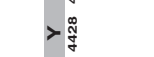 & 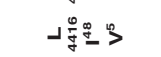 & o票 & \\
\hline 《旁 & -禀> & 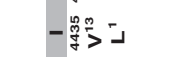 & 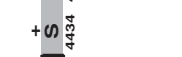 & 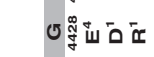 & $0 \stackrel{0}{0}$ & $x_{\frac{\mathrm{g}}{g}}^{\circ}$ & \\
\hline$>$ 高- & •-淼下下 & 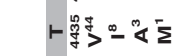 & 崔 & ৩すั & 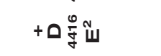 & ৩。্⿳亠口冋丁口 & \\
\hline >兽里 & צ尊 & 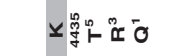 & $>$ 释 & 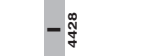 & $x^{\circ} \frac{0}{q} \bar{x}$ & $>\frac{\sigma}{\sigma}$ & \\
\hline 品嗙然的 & 尺 哓山 & $\stackrel{\circ}{\stackrel{0}{F}>\frac{\mathscr{q}}{q}}$ & 品>蓆 & $\stackrel{\circ}{\stackrel{9}{9} \dddot{q}}$ & 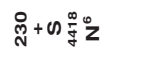 & 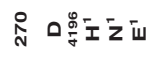 & \\
\hline 喁 & 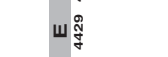 & Q尊 & 㧹 & ৩ঞ & ○旁 & 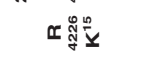 & \\
\hline 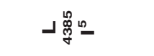 & $+\lrcorner \hat{q} \bar{y}$ & $3 \frac{\circ}{q}$ & *0军 & 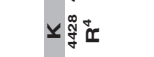 & $\alpha \frac{\circ}{q}$ & 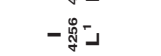 & \\
\hline$z_{\bar{q}}^{\bar{g}}$ & I & 曲尊 & *⿻口最 & 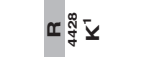 & 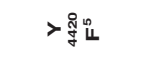 & 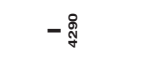 & \\
\hline 峿 & *トサ ஜ & 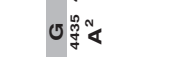 & *0骂 & $צ \underset{q}{\mathbb{q}}$ & $+>\nsubseteq$ & $\mathbf{x}_{\text {章 }}$ & \\
\hline 羉 & 啳 & 㧹 & 噂 & แ罗 & $>\underset{q}{q}$ & 《ซ⿱乛⿻丷十 & \\
\hline 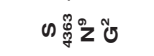 & 回路 & 橧 & $z \frac{\mathscr{q}}{q}$ & $z \nsubseteq$ & $\llbracket \stackrel{q}{q}$ & 骂 & \\
\hline 《鸹》 & \lrcorner 彝 & Y量的 & $*>\frac{q}{q}$ & +工災 & 山寽 & $+\infty$ 婁 & \\
\hline$\sum \hat{z}=$ & 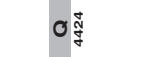 & 增 & $a \frac{⿱ o}{q}$ & 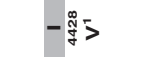 & $Z \bar{q} \bar{Y} \bar{I}$ & ๔䨌 & \\
\hline 《承的 & 3茮 & 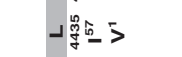 & -骂 & 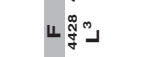 & 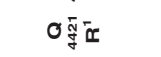 & Q昱 & \\
\hline 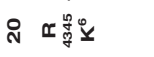 & $8-\frac{\tilde{z}}{\tilde{N} \Sigma} \bar{\Sigma}$ & 号 山尊品 & 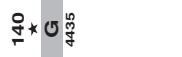 & $\stackrel{\infty}{\sim}>\frac{\circledast}{q}$ & \ิ & 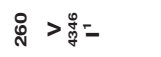 & \\
\hline 3寻 & 啳 & $>$ 尊正 & 嗙 & $\varangle \mathbb{\pi}_{\frac{\pi}{q}}$ & צ⿻ำ & $>$ 虽- & \\
\hline$z_{\frac{F}{\sigma}}^{*}$ & $2 \underset{z}{q}$ & 嶟 & +山事 & $\Sigma \Sigma_{\tilde{q}}^{\mathbb{q}}$ & 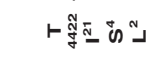 & $x_{\frac{\bar{g}}{\sigma}}$ & \\
\hline 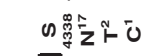 & 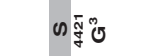 & +十鲂命 & 噂 & o导 & $-\frac{z}{z}>$ & - 高 & \\
\hline 国㪺 & 啋 & 㰘骂 & 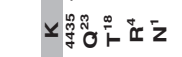 & $>$ & 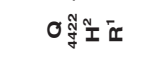 & 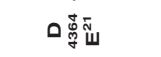 & \\
\hline$>$ 爱 & 唼 & +o㝵 & -曾塄 & $\varangle \frac{\pi}{q}$ & Х读的 & 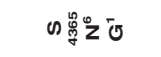 & \\
\hline 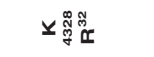 & $+>\frac{1}{q}=$ & 噂 & 㧹是的 & ト䝉 & o ฐ & 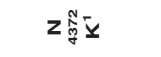 & \\
\hline 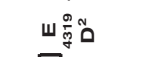 & 㖕 & ト尊 & 㧹 & 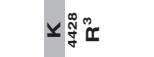 & د齐 & 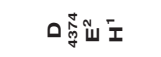 & \\
\hline 国湿 & 时 & *㹎 & 3喿 & - & سสูจष & o 号 & \\
\hline 骀总墕 & +工喿 & 嬉下的 & 3 喿 & I羿 0 & 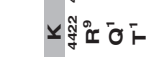 & 一鲁」 & \\
\hline 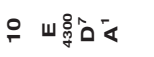 & 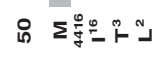 & 吅高的 & 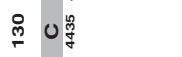 & 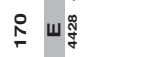 & $\stackrel{\circ}{\frac{N}{N}}$ & 品> & \\
\hline O: & 《得 & 一萦 & 嶟 & 《釉 & ơ⿱ & $>$ 京 & \\
\hline 《亭 & 山守 & $>$ 索二 & • <桑下 & o & 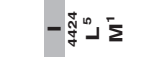 & 《余 & 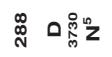 \\
\hline 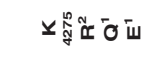 & $\sigma^{\frac{⿱}{q}}$ & 嵉 & 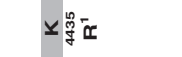 & ه & 口然山 & 琼 & ш ㅇํํ \\
\hline 口 đู๊ 的 $\bar{z}$ & $\Upsilon^{\circ}$ & 嗦 & 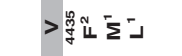 & 风吿 & ト絭芯 & $山 \frac{N}{q}$ & 口罵 \\
\hline$-\frac{\bar{\sigma}}{\sigma}$ & 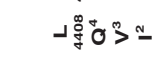 & 罸 & 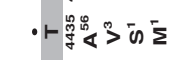 & 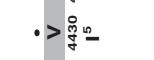 & 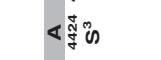 & 唩 & 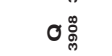 \\
\hline vढ্⿳亠口冋̆ & 梌 & 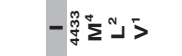 & 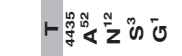 & 嘌 & - & $Y_{\frac{p}{q}}^{\frac{m}{\alpha}}$ & 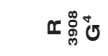 \\
\hline ه & 唯 & $>$ > & 的累 & 嘌出 & - & $3 \frac{\pi}{\sigma}$ & 的尊 \\
\hline 」亳 & 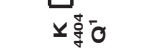 & 噂 & ト旁品 & -孚 & 口导 & 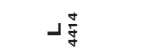 & $\varangle_{\%}^{\circ}$ \\
\hline 山兽 & 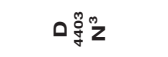 & 㗪 & *㹡 & - & •>染名 & -紊 & $>$ 高满 \\
\hline
\end{tabular}


Among the 75 heavily treated patients, 67 eventually achieved or maintained complete virologic suppression for $\geq 6$ months (median 20 months) while receiving a Raltegravircontaining regimen, two patients experienced virological failure with the development of raltegravir resistance, and six did not remain on raltegravir long enough to be evaluated. The two patients who subsequently experienced virological failure on a raltegravir-containing regimen had no evidence major or minor INI-resistance mutations in the samples sequenced for this study.

\section{Updated Analysis of HIV-1 IN Polymorphism in INI-Naïve Patients}

In 2008, we examined IN sequences from 1563 INI-naive patients and reported that no major mutations occurred as natural variants. ${ }^{10}$ However, several mutations associated with slight reductions in INI susceptibility did occur at low levels. With the current availability of IN sequences from 4320 INI-naiive patients, we chose to repeat this analysis to reassess the reliability of our earlier findings. For this analysis, we included data from several recently published studies with large numbers of IN sequences from INI-naïve patients. ${ }^{11-15}$

We combined the IN sequences of the 150 patients we describe here with sequences from 4170 previously reported INInaïve patients published in GenBank as of April 15, 2010 to calculate the frequency with which each mutation has been reported at each IN position. Figure 1 shows the proportions of all mutation present in $\geq 0.5 \%$ of the 4470 INI-naïve patients. Among the sequences from these 4320 patients, $48 \%$ belonged to subtype B, $16 \%$ to CRF01-AG, $12 \%$ to subtype C, $8 \%$ to $\mathrm{CRF} 01 \mathrm{AE}, 5 \%$ to subtype $\mathrm{A}$, and $11 \%$ to other subtypes and CRFs. These proportions differ from our previous analysis of 1563 patients of whom $25 \%$ had subtype B viruses, $6 \%$ had CRF01-AG viruses, and $29 \%$ had subtype $C$ viruses.

Of the 288 IN positions, $41 \%$ (117) had one or more mutations present at a proportion $\geq 0.5 \% ; 59 \%$ of positions were nonpolymorphic. The proportion of polymorphic residues is nearly identical to the proportion we reported 2 years earlier in which $40 \%$ (115) of IN positions had mutations present at a proportion $\geq 0.5 \%$. In addition, the total number of polymorphisms increased from 177 (a mean of 1.5 polymorphisms per polymorphic position) in our previous analysis to 192 in our current analysis (a mean of 1.6 polymorphisms per polymorphic position). The mean coefficient of variation of mutation prevalence between the two datasets was $0.29 \%$. None of the major INI-resistance mutations was found to be polymorphic in either analysis. Nor were their statistically significant changes at any of the minor INI-resistance mutations-H51Y, L74M, T97A, E138AK, S153Y, E157Q, G163KR, $\mathrm{S} 230 \mathrm{R}$, and R263KR - each of which occurred at a prevalence $\leq 2.0 \%$ in INI-naïve patients.

\section{Acknowledgments}

$\mathrm{VV}, \mathrm{MB}$, and RWS were supported in part by NIAIDAI46148-01 and a research grant from Merck Laboratories. The sequences reported in this study are being submitted to the GenBank under the accession numbers HM450153HM450302.

\section{Author Disclosure Statement}

No competing financial interests exist.

\section{References}

1. Goldgur Y, Craigie R, Cohen GH, et al.: Structure of the HIV1 integrase catalytic domain complexed with an inhibitor: A platform for antiviral drug design. Proc Natl Acad Sci USA 1999;96:13040-13043.

2. Chen JC, Krucinski J, Miercke LJ, et al.: Crystal structure of the HIV-1 integrase catalytic core and C-terminal domains: A model for viral DNA binding. Proc Natl Acad Sci USA 2000;97:8233-8238.

3. Wang JY, Ling H, Yang W, and Craigie R: Structure of a two-domain fragment of HIV-1 integrase: Implications for domain organization in the intact protein. EMBO J 2001;20: 7333-7343.

4. Alian A, Griner SL, Chiang V, et al.: Catalytically-active complex of HIV-1 integrase with a viral DNA substrate binds anti-integrase drugs. Proc Natl Acad Sci USA 2009; 106:8192-8197.

5. Low A, Prada N, Topper M, et al:: Natural polymorphisms of human immunodeficiency virus type 1 integrase and inherent susceptibilities to a panel of integrase inhibitors. Antimicrob Agents Chemother 2009;53:4275-4282.

6. Myers RE and Pillay D: Analysis of natural sequence variation and covariation in human immunodeficiency virus type 1 integrase. J Virol 2008;82:9228-9235.

7. Ceccherini-Silberstein F, Malet I, D'Arrigo R, Antinori A, Marcelin AG, and Perno CF: Characterization and structural analysis of HIV-1 integrase conservation. AIDS Rev 2009; 11:17-29.

8. Bushman FD and Miller MD: Tethering human immunodeficiency virus type 1 preintegration complexes to target DNA promotes integration at nearby sites. J Virol 1997;71:458-464.

9. de Oliveira T, Deforche K, Cassol S, et al.: An automated genotyping system for analysis of HIV-1 and other microbial sequences. Bioinformatics 2005;21:3797-3800.

10. Rhee SY, Liu TF, Kiuchi M, et al.: Natural variation of HIV-1 group $M$ integrase: Implications for a new class of antiretroviral inhibitors. Retrovirology 2008;5:74.

11. Garrido C, Geretti AM, Zahonero N, et al.: Integrase variability and susceptibility to HIV integrase inhibitors: Impact of subtypes, antiretroviral experience and duration of HIV infection. J Antimicrob Chemother 2010;65:320-326.

12. Eshleman SH, Hudelson SE, Smith $\mathrm{P}$, et al.: Analysis of pol integrase sequences in diverse HIV type 1 strains using a prototype genotyping assay. AIDS Res Hum Retroviruses 2009;25:343-345.

13. van Hal SJ, Herring B, Deris Z, Wang B, Saksena NK, and Dwyer DE: HIV-1 integrase polymorphisms are associated with prior antiretroviral drug exposure. Retrovirology 2009;6:12.

14. Brennan CA, Stramer SL, Holzmayer V, et al.: Identification of human immunodeficiency virus type 1 non-B subtypes and antiretroviral drug-resistant strains in United States blood donors. Transfusion 2009;49:125-133.

15. Sichtig N, Sierra S, Kaiser R, et al.: Evolution of raltegravir resistance during therapy. J Antimicrob Chemother 2009;64:25-32.

Address correspondence to: Vici Varghese

Grant Bldg Room S-146

Division of Infectious Diseases

Department of Medicine Stanford University 300 Pasteur Drive Stanford CA 94305

E-mail: viciv@stanford.edu 